\title{
Fast X-ray Oscillations During Magnetar Flares
}

\author{
Tod E. Strohmayer \\ Astrophysics Science Division, NASA's Goddard Space Flight Center, Greenbelt, MD
}

\begin{abstract}
.
The giant flares produced by highly magnetized neutron stars, "magnetars," are the brightest sources of high energy radiation outside our solar system. Serendipitous observations with NASA's Rossi X-ray Timing Explorer (RXTE) of the two most recent flares resulted in the discovery of high frequency oscillations in their X-ray fluxes. The frequencies of these oscillations range from $\approx 20 \mathrm{~Hz}$ to as high as $1800 \mathrm{~Hz}$, and may represent the first detection of global oscillation modes of neutron stars. Here I will present an observational and theoretical overview of these oscillations and discuss how they might allow us to probe neutron star interiors and dense matter physics.
\end{abstract}

Keywords: neutron stars, torsional oscillations, pulsars, rotation, magnetospheres

PACS: $97.10 . \mathrm{Sj}, 97.60 . \mathrm{Jd}, 95.85 . \mathrm{Nv}$

\section{INTRODUCTION}

The existence of young $\left(<10^{4}\right.$ yrs $)$ neutron stars with magnetic fields upwards of $10^{15} \mathrm{G}$, "magnetars," is now widely accepted. Historically, two classes of neutron stars had been associated with such strong magnetic fields, the Soft gamma-ray repeaters (SGRs), and the Anomalous X-ray Pulsars (AXPs). The former were first recognized as sources of super-Eddington, soft gamma-ray bursts, while the latter were revealed as slowly spinning, rapidly braking pulsars emitting much more X-ray flux than could be accounted for by their spin-down energy alone. In each case the energy associated with the phenomena was attributed to decay of a super-strong magnetic field. In recent years the distinctions between the two classes have steadily narrowed; for example, SGRs are now also known to be "anomalous" pulsars, and AXPs have been observed to burst (see Woods \& Thompson 2006 for a recent review of magnetars).

One distinction that remains is that the SGRs are, so far, the only known sources of giant flares (also known as hyper-flares). These are the brightest cosmic events originating outside the solar system, in terms of flux received at Earth. They are characterized by an intense gamma-ray spike lasting tenths of seconds, and reaching luminosities of $10^{44-46}$ ergs $\mathrm{s}^{-1}$. Only three have been observed to date, with the first being the famous March 5, 1979 gamma-ray flare from SGR 0526-66 in the LMC (Mazets et al. 1979). The two most recent giant flares; from SGR 1900+14 in 1998 August (Hurley et al. 1999), and SGR 1806-20 in 2004 December (Palmer et al. 2005), are the subject of this work.

Recent high time resolution studies of these flares using data from the Proportional Counter Array (PCA) onboard the Rossi X-ray Timing Explorer (RXTE) have resulted in the discovery of a new phenomenon associated with these events. Both flares produced fast, rotation-phase-dependent X-ray oscillations in the $20-150 \mathrm{~Hz}$ range, and the 2004 flare additionally produced $\mathrm{kHz}$ oscillations in the $625-1,800 \mathrm{~Hz}$ range. Israel et al. (2005) first reported the discovery of $\approx 18,30$, and $90 \mathrm{~Hz}$ quasi-periodic oscillations 



FIGURE 1. Examples of QPOs detected during the magnetar flares from SGR 1900+14 (left) and SGR 1806-20 (right). QPOs in SGR 1900+14 are evident in an average power spectrum computed from rotational phases centered on the phase at which an $84 \mathrm{~Hz}$ QPO was first detected (upper traces), while no QPOs are seen at other phases (bottom trace). QPOs at 30 and $92 \mathrm{~Hz}$ are strongly detected in this average power spectrum from the interpulse phase in the SGR 1806-20 flare (right). After Strohmayer \& Watts (2005, 2006), respectively.

(QPO) in the December, 2004 event, and suggested that the 30 and $90 \mathrm{~Hz}$ QPOs could be linked with seismic (torsional) vibrations of the neutron star crust. Strohmayer \& Watts (2005) then reported the discovery of a sequence of QPOs in the SGR 1900+14 event. They found a set of frequencies; 28, 53.5, 84 , and $155 \mathrm{~Hz}$, that could consistently be associated with a sequence of low $l$ toroidal modes (denoted $l_{0}$ ) of the elastic neutron star crust (see, for example, Hansen \& Cioffi 1980; McDermott, van Horn \& Hansen 1988; Duncan 1998; Piro 2005). In both flares the oscillations are episodic, that is, their amplitudes vary considerably with time and rotational phase. Watts \& Strohmayer (2006) also examined Ramaty High Energy Solar Spectroscopic Imager (RHESSI) data from the SGR 1806-20 event, confirmed the presence of the $18 \mathrm{~Hz}$ and $90 \mathrm{~Hz}$ QPOs, and found evidence for additional oscillations at $26 \mathrm{~Hz}$ and $626 \mathrm{~Hz}$. Most recently, Strohmayer \& Watts (2006) reexamined the RXTE data from the SGR 1806-20 event, and found additional oscillations at 150,625, and 1,840 Hz. Figure 1 shows examples of QPO detections in each object.

\section{KEY PROPERTIES OF THE QPOS}

The similar phenomenology of the oscillations in the two sources, as well as the closeness of some of the measured frequencies argues convincingly that we are seeing the same physical process in each case. The connection with torsional modes of the crust seems plausible for several reasons; 1) The observed frequencies are consistent with theoretical expectations for such modes, and can be more or less self-consistently associated with a sequence of modes with varying spherical harmonic index, $l$. The higher frequency QPOs (above $600 \mathrm{~Hz}$ ) can plausibly be interpreted as modes with at least one node in the radial displacement eigenfunction. 2) The magnetic instability which pow- 
ers the flares will very likely fracture the neutron star crust, generating seismic motions within the star (Flowers \& Ruderman 1977; Thompson \& Duncan 1995; Duncan 1998; Thomson \& Duncan 2001; Schwartz et al. 2005). 3) The strong rotational phase dependence argues for a mechanism associated with particular sites on the stellar surface, such as a fracture zone or magnetic field bundle. 4) Mechanical motions provide a natural explanation for the relatively high coherence of the oscillations. 5) Horizontal motions of the crust could modulate the beaming pattern associated with the strong magnetic field, providing a mechanism to modulate the X-ray flux. Moreover, beaming can act as an "amplifier," producing potentially large X-ray modulations from modest horizontal displacements. Although the present evidence for torsional modes is very suggestive, it is not yet definitive. Levin (2006), for example, has argued that toroidal modes may damp too quickly to account for the detection of oscillations some minutes after the onset of the flare. In addition some of the detected frequencies $(18 \mathrm{~Hz}, 26 \mathrm{~Hz})$ do not fit easily into current torsional mode models, without invoking magnetic splitting or other complications, such as coupling of the crust with the core.

\section{TORSIONAL MODE INTERPRETATION}

Since the detections of the magnetar QPOs there has been a substantial theoretical effort to interpret the observed frequencies, amplitudes and other properties. As mentioned above, the initial suggestions focused on oscillations of the neutron star crust, the so called torsional modes, denoted ${ }_{l} t_{n}$, where $l$ is the spherical harmonic index for the mode, and $n$ is the number of radial nodes in the eigenfunction (Israel et al. 2005; McDermott, Van Horn \& Hansen 1988). These modes sense the shear wave speed in the crust, and its size (see, for example, Strohmayer et al. 1991; Duncan 1998). These quantities depend on the global structure of the star and hence the equation of state (EOS) of matter in the deep interior (see Strohmayer \& Watts 2005; Lattimer \& Prakash 2007). Note also that the strong magnetic field can boost the "tension" in the crust and thus modify the mode periods, perhaps non-isotropically (see Duncan 1998; Messios, Papadopolous \& Stergioulas 2001). Recently, Samuelsson \& Andersson (2007) have explored torsional mode oscillations in neutron stars using a general relativistic formulation with neglect of the metric perturbations (the so called Cowling approximation). They derive a set of analytic estimates for the mode frequencies based on numerical calculations, and estimate the frequency (in $\mathrm{Hz}$ ) of the $n=0$ modes to be

$$
f\left({ }_{l} t_{0}\right)=27.65 R_{10}^{-1} \frac{((l-1)(l+2))^{1 / 2}}{2} \frac{\left(\left(1.705-0.705 \beta_{*}\right)\left(0.1055+0.8945 \beta_{*}\right) \beta_{*}\right)^{1 / 2}}{\left(1.0331 \beta_{*}-0.0331\right)},
$$

where $R_{10}=R / 10 \mathrm{~km}$ and $\beta_{*}=\beta / 0.2068$, and $\beta=G M / c^{2} R$ (for $R_{10}=1$ and $M=$ $1.4 M_{\odot}, \beta_{*}=1$ ). The expression for the $n>0$ modes (again, in $\mathrm{Hz}$ ) is

$$
f\left({ }_{l} t_{n}\right)=1107.3 \frac{n\left(0.1055+0.84945 \beta_{*}\right)}{R_{10}} \frac{\beta_{*}}{1.0166 \beta_{*}-0.0166} .
$$

These expressions do not include any correction for magnetic field effects. Note that the scaling with $l$ here is slightly different than the $l(l+1)$ scaling in previous estimates 

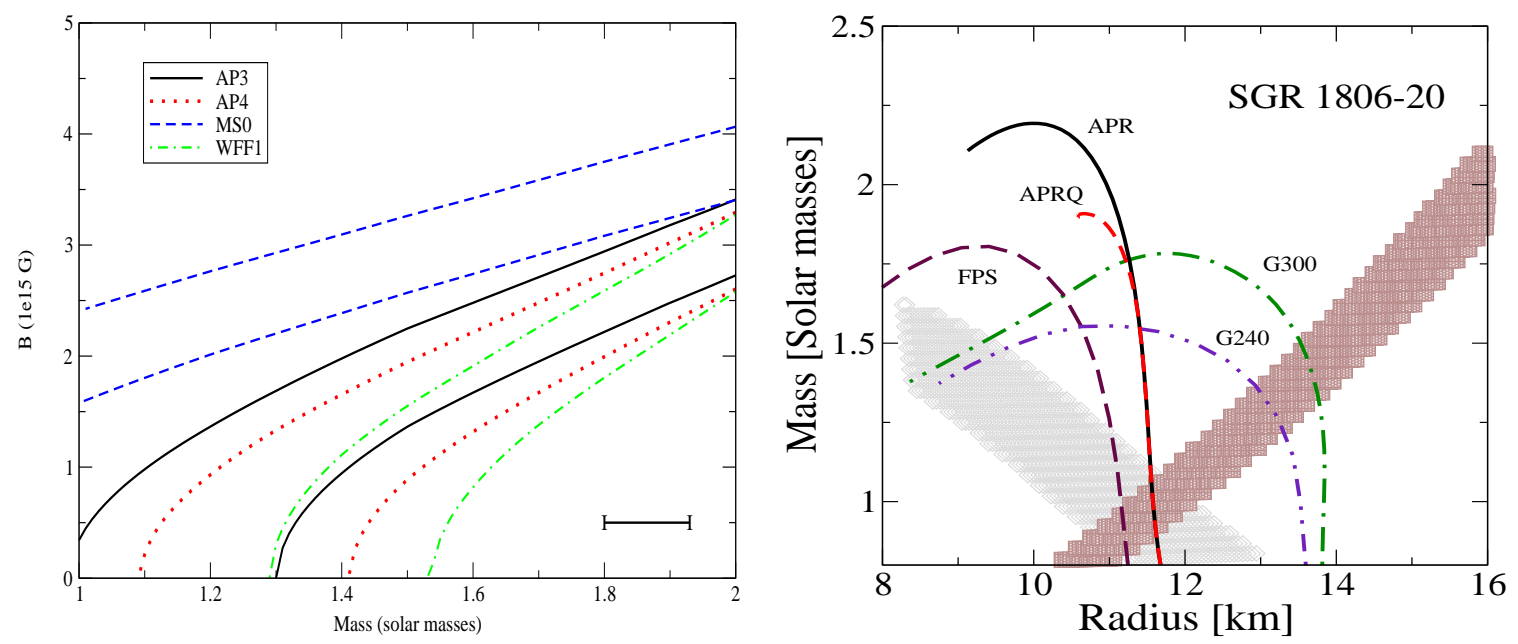

FIGURE 2. Constraints on neutron star structure from magnetar QPOs. The stellar mass and magnetic field required to give the ${ }_{2} t_{0}$ mode frequencies inferred for SGR 1806-20 (30.4 Hz), and SGR 1900+14 $(28 \mathrm{~Hz})$ are shown in the left panel (after Strohmayer \& Watts 2005) for several different EOS models. For each EOS, two lines are shown. The upper line is for SGR 1806-20, and the lower line for SGR 1900+14. The regions allowed based on $n=0$ and $n=1$ mode identifications in SGR 1806-20 of Samuelsson \& Andersson (2007) are shown in the right panel (after Samuelsson \& Andersson 2007). The shaded region with decreasing mass versus radius denotes the $n=0$ constraints. The orthogonal nature of the constraints for $n=0$ and $n>0$ modes clearly demonstrates the potential power of detecting both types of modes.

(see Duncan 1998, Piro 2005). This can lead to somewhat different mode assignments for particular observed frequencies. Since these relations depend on both the stellar mass and radius they are clearly EOS dependent, and thus secure mode identifications could provide constraints on the EOS. However, the effects of the magnetic field on the oscillation mode spectrum will likely have to be understood quantitatively before precise EOS constraints will be possible. Nevertheless, the promise of using asteroseismology to constrain the neutron star EOS is undeniable.

To illustrate the possibilities we review two examples from the recent literature. Strohmayer \& Watts (2005) tabulated the stellar parameters that give ${ }_{2} t_{0}$ oscillations at $28 \mathrm{~Hz}$ (SGR 1900+14) and $30.4 \mathrm{~Hz}$ (SGR 1806-20). These were the fundamental mode identifications that they made based on the mode periods, including an isotropic magnetic correction, estimated by Duncan (1998). Figure 2 (left) shows the results for four different EOS discussed in Lattimer \& Prakash (2001). The results suggest that if the stars have similar magnetic field strengths, their masses must differ by more than $0.2 M_{\odot}$. Since the masses of radio pulsars (a young neutron star population) have been found to be consistent with a fairly narrow Gaussian distribution, $M=1.35 \pm 0.04 M_{\odot}$, a perhaps more likely scenario is that the stars have similar masses but different magnetic field strengths. If both stars have masses $\approx 1.35 M_{\odot}$, then it is difficult to account for both frequencies with the softest EOS (WFF1); and the stiffest EOS (MS0) predicts magnetic fields for both systems that are far larger than those inferred from timing studies (Woods et al. 2002). The moderately stiff EOSs AP3 and AP4 can account for the observed frequencies, and give magnetic field strengths that agree reasonably well with those derived from timing measurements of both stars (Woods \& Thompson 
2006). Samuelsson \& Andersson (2007) used their relativistic formulation to determine the range of stellar masses and radii which could produce acceptable matches to the observed frequencies for some sequence of $l$ values. Their results for SGR 1806-20 are also shown in Figure 2 (right). They are able to associate the $\approx 29,93$, and $150 \mathrm{~Hz}$ QPOs with $l=2,6$ and 10 modes (all with $n=0$ ). The higher frequency QPO at 626 $\mathrm{Hz}$ is associated with an $n=1$ mode for which $l$ is not greatly constrained. The allowed regions for the $n=0$ and $n=1$ modes are largely orthogonal, and in principle, could provide very tight constraints on the stellar parameters.

\section{REMAINING THEORETICAL CHALLENGES}

\section{Crust - Core Coupling}

The question of whether or not pure crust models are adequate is an important one. Levin (2006) argued that if there is a strong perpendicular magnetic field threading the crust - core boundary, then large horizontal displacements at the base of the crust must excite vibrations in the core, perhaps significantly damping crust motions. The effects of coupling may, however, be mitigated in several ways. For example, coupling to the core will depend sensitively on the amplitude at the boundary, and previous calculations show that this amplitude can be substantially smaller than that at the top of the crust (see McDermott et al. 1988, for example). The physics at the boundary layer at the base of the crust will also be crucial (see, for example, Kinney \& Mendell 2003). The elastic properties at the base of the crust (Pethick \& Potekhin 1998) have not yet been considered, and could substantially modify the eigenfunctions in this region. In addition, coupling will likely depend rather sensitively on both the magnetic field geometry and the particular displacement pattern of individual modes. The modes that persist, and that we observe, may be those for which the coupling is minimal. The presence of a strong toroidal field in the core of the star could also reduce coupling by making the core more rigid and less prone to excitation.

If the coupling between crust and core is strong, by virtue of boundary layer physics or magnetic field threading, one needs to consider the global magneto-elastic modes of the neutron star. As first suggested by Israel et al. (2005), global modes can accommodate the lower frequency 18 and $26 \mathrm{~Hz}$ QPOs very easily. A recent paper by Glampedakis et al. (2006) developed a simple slab model of global magneto-elastic oscillations that showed two interesting features. Firstly, it confirmed the presence of modes at lower frequencies than the "pure crust" toroidal modes. Secondly, the model exhibited modes for which the amplitudes in the crust were strong; in these cases the frequency was very close to the well-established "pure crust" frequencies. In other words, even with coupling included, it is possible to obtain very similar frequencies to those that we know match the data. Although the model was very simple (slab geometry, for example, is not adequate to describe behavior deep in the core), this gives us some indication that global modes may have similar frequencies.

In a more recent calculation, Levin (2007) has explored in some detail the coupling between normal modes in the crust and an MHD continuum in the fluid core, in the context of a uniform magnetic field and thin spherical crust. He finds that the torsional 


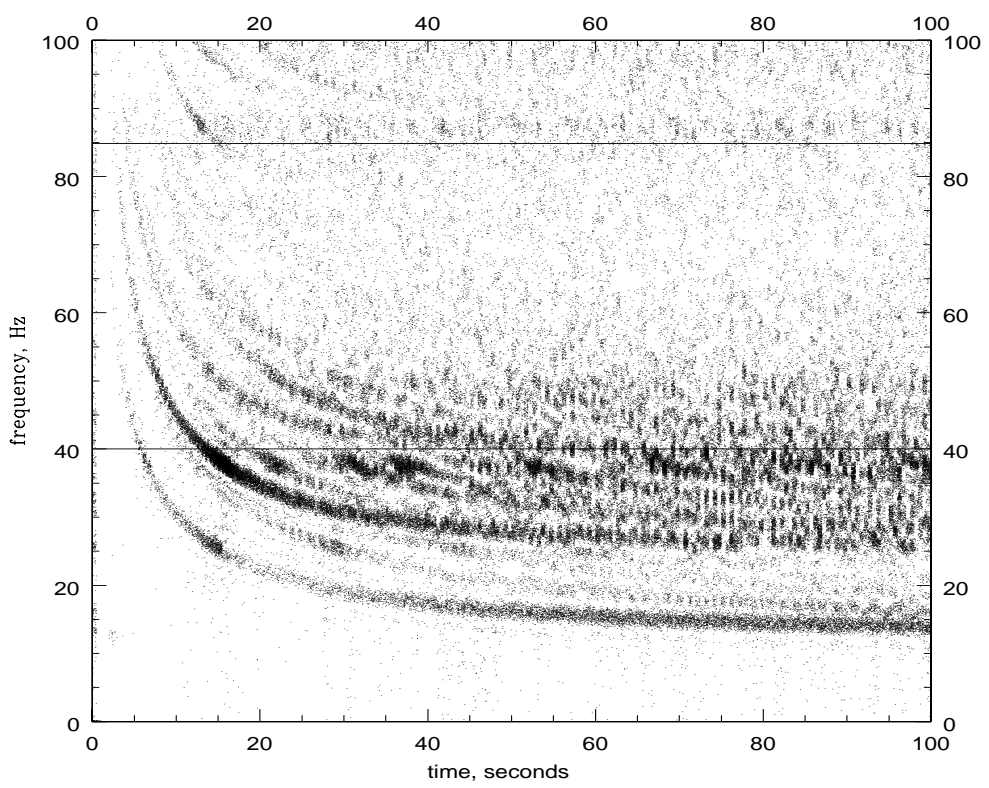

FIGURE 3. A dynamic power spectrum computed from a physical simulation of the crustal displacement in a magnetar, including the interaction between crust and core, is shown (after Levin 2007). The density of points tracks the Fourier power. The low frequency QPOs asymptotically approach the MHD core continuum turning points (see Levin 2007 for a detailed discussion). Interestingly, excess oscillation power also appears near the frequencies of crustal torsional modes (horizontal lines).

modes in the crust quickly exchange energy with the core MHD continuum, and that there are natural QPOs associated with the dynamics of such a system. The QPOs are associated with either the "edges" of the continuum, or at so called "turning points," where there is a local extremum in the frequency distribution of the continuum. Levin describes a relatively simple, but physically well motivated toy model in which a large number of small pendula are coupled to a single large pendulum. The frequency of the large pendulum lies in the middle of the range of the smaller ones. In this simulation the big pendulum represents an initially stressed magnetar crust, and the small pendula represent the MHD core continuum with which it is coupled. After release, the motion of the big pedulum is rapidly damped, as energy is drained into the smaller pendula. In this system, Levin finds QPOs at the edges of the continuum, that is, at the lowest and highest frequencies of the little pendula. The QPOs occur at these frequencies, because it is only in the vicinity of these points where the pulling of individual small pendula on the large one are not cancelled out by other small pendula. While the real magnetar dynamics are much more complex, Levin argues, rather convincingly, that this simple model captures much of the relevant physics. In a more realistic simulation of the crust - core coupling he finds interesting qualitative agreement with many of the observed properties of the magnetar flares. In particular, strong QPOs at about $18 \mathrm{~Hz}$ are present and would appear to be consistent with the turning point of the MHD core continuum. We show in Figure 3 the dynamic power spectrum from Levin's calculation, and the QPOs are clearly evident as the darker bands in the spectrum, particularly at 
the lower frequencies $(18-30 \mathrm{~Hz})$. Moreover, QPOs appear to be excited or amplified at frequencies near the pure crustal mode frequencies. Interestingly, the QPOs in these simulations also show a clear drifting in frequency with time which remains to be clearly understood. While this initial theoretical work appears very promising, there are still many remaining questions, and more realistic physical scenarios need to be addressed, including more realistic magnetic field geometries. More work in this area is clearly warranted.

\section{Magnetospheric Coupling: X-ray Modulation}

While the evidence is now substantial that the magnetar oscillations are due to neutron star vibrations, there is still a great deal of uncertainty on the details of how stellar surface motions get translated into rather strong modulations in the observed X-ray flux. Moreover, the present data hint at a complex temporal evolution of the oscillations. For example, some of the oscillations are seen throughout the flare, others are detected only half way through the tail, and in general the highest frequency modes appear to be rather short-lived. This, and other, complexities require explanation, and to do so we must understand the physics of mode excitation, damping, and X-ray modulation.

It has been suggested that the "shaking" of the magnetic field lines due to stellar vibrations, coupled with strong beaming of the emitted radiation, could play an important role in the modulation mechanism (see Strohmayer \& Watts 2006). Recently, Timokhin, Eichler \& Lyubarsky (2007) have proposed that modulation of the X-ray flux is linked to variations of the magnetospheric currents induced by oscillatory motions of the surface (via the magnetic field). They show that the angular distribution of the optical depth to resonant Compton scattering produced by a particular torsional mode pattern can be highly anisotropic, and argue that this can account for the observed rotational phase dependence of the oscillations. Moreover, they estimate the amplitude of surface motions required to produce the observed oscillation amplitudes, and find that this is about $1 \%$ of the stellar radius, or about 100 meters. This amplitude represents a substantial amount of mechanical energy, Timokhin et al. (2007) estimate $\approx 10^{42}$ ergs per mode would need to be deposited in crustal motions by the giant flares. A significant amount of this energy would likely end up as heat in the crust, and could power some of the long term Xray afterglows that have been observed (Kouveliotou et al. 2003). A nice feature of this model is that it fits well with an emerging consensus that the hard X-ray emission from magnetars is associated with scattering by magnetospheric currents (Lyutikov \& Gavriil 2006; Fernández \& Thompson 2007). However, more realistic, quantitative predictions will be required to make detailed comparisons with observations.

\section{CONCLUSIONS}

Serendipitous RXTE observations of the magnetar flares from SGR 1806-20, and SGR $1900+14$ indicate that a complex pulsation phenomenology is associated with these events. The discovery of new kHz-range frequencies consistent with theoretical predictions for $n>0$ torsional modes provides strong evidence that we may in fact be seeing 
vibration modes of the neutron star crust excited by these catastrophic events. If this is true, then it opens up the exciting prospect of probing the interiors of neutron stars in a manner analogous to helioseismology. Additional excitement is warranted when we consider that all the current datasets used to explore these oscillations have been purely serendipitous. That is, they have not been optimized in any way for studying these signals. This suggests that a wealth of additional information would likely be found from instruments better optimized to capture with high time resolution the flood of X-rays produced by these events. In order to fully exploit such observations more theoretical work is definitely needed to enable more accurate mode identifications, to better understand the excitation and damping mechanisms of modes and how they can couple to the $\mathrm{X}$-ray emission, and to make more precise inferences on neutron star structure.

\section{REFERENCES}

1. Duncan, R.C. 1998, ApJ, 498, L45

2. Fernández, R., \& Thompson, C. 2007, ApJ, 660, 615

3. Flowers, E., \& Ruderman, M.A. 1977, ApJ, 215, 302

4. Glampedakis, K., Samuelsson, L. \& Andersson, N. 2006, MNRAS, 377, 630

5. Hansen, C. J. \& Cioffi, D. F. 1980, ApJ, 238, 740

6. Hurley, K., et al., 1999, Nature, 397, 41

7. Israel, G., et al., 2005, ApJ, 628, L53

8. Kinney, J.B., \& Mendell, G. 2003, Phys. Rev. D, 67, 024032

9. Kouveliotou, C., et al. 2003, ApJ, 596, L79

10. Lattimer, J. M., \& Prakash, M. 2007, Phys. Rep., 442, 109

11. Lattimer, J. M. \& Prakash, M. 2001, ApJ, 550, 426

12. Levin, Y. 2007, MNRAS, 377, 159

13. Levin, Y. 2006, MNRAS, 368, L35

14. Lyutikov, M., \& Gavriil, F. P. 2006, MNRAS, 368, 690

15. Mazets, E. P. et al. 1979, Observations of a flaring X-ray pulsar in Dorado, Nature, 282, 587

16. McDermott, P.N., van Horn, H.M., \& Hansen, C.J. 1988, ApJ, 325, 725

17. Messios, N., Papadopoulos, D.B., Stergioulas, N., 2001, MNRAS, 328, 1161

18. Palmer, D.M., et al., 2005, Nature, 434, 1107

19. Pethick, C.J. \& Potekhin, A.Y. 1998, Phys. Lett. B, 427, 7

20. Piro, A.L. 2005, ApJ, 634, L153

21. Samuelsson, L., \& Andersson, N. 2007, MNRAS, 374, 256

22. Schwartz, S.J., et al. 2005, ApJ, 627, L129

23. Strohmayer, T.E., \& Watts, A.L. 2006, ApJ, 653, 593

24. Strohmayer, T.E., \& Watts, A.L. 2005, ApJ, 632, L111

25. Strohmayer, T., van Horn, H. M., Ogata, S., Iyetomi, H., \& Ichimaru, S. 1991, ApJ, 375, 679

26. Thompson, C., \& Duncan, R.C. 2001, ApJ, 561, 980

27. Thompson, C., \& Duncan, R.C. 1995, MNRAS, 275, 255

28. Timokhin, A. N., Eichler, D., \& Lyubarsky, Y. 2007, ArXiv e-prints, 706, arXiv:0706.3698

29. Watts, A.L. \& Strohmayer, T.E. 2006, ApJ, 637, L117

30. Woods, P.M., Thompson, C., 2006, Soft Gamma Repeaters and Anomalous X-ray Pulsars: Magnetar Candidates, in Compact Stellar X-ray Sources, eds. W.H.G. Lewin and M. van der Klis, Cambridge University Press

31. Woods, P.M., et al., 2002, ApJ, 576, 381 